increase in prevalence of ASD have included more inclusive definitions, improved recognition of the symptoms, greater parental concern, and possible epigenetic factors and modifications of gene expression. The theory that the measles-mumps-rubella (MMR) vaccine and thimerosol were responsible for the apparent increased prevalence of autism has largely been put to rest (Madsen KM et al. Pediatrics 2003;112:604-606; Smeeth L et al. Lancet 2004;364:963-969). ASD has a strong genetic basis, and a specific medical cause or environmental trigger is found in only $6-10 \%$ of cases, eg. prenatal insult, metabolic and toxic disorders, tuberous sclerosis, and postnatal encephalitis (Baird G et al. BMJ 2003;327:488-493; see Ped Neur Briefs October 2003;17:79-80). The study of the etiology of autism is multifaceted, and future research will involve pediatric neurologist, psychiatrist, pathologist, and geneticist.

Oculomotor studies in autism. Pursuit eye movement deficits were observed in a study of 60 high-functioning autistic young adults compared to 94 normal control subjects, at the University of Illinois, Chicago (Takarae Y et al. Brain Dec 2004;127:2584-2594). The authors conclude that their findings are consistent with reduced functional connectivity within the visual pursuit system caused by abnormalities in brain maturation in autism.

\title{
BROCA'S AREA ASYMMETRY AND LANGUAGE DEFICITS IN AUTISM
}

The asymmetry of frontal language cortex in boys with autism, previously reported, was investigated further in a sample of 22 boys with autism compared to 9 boys with specific language impairment (SLI) and 11 normal controls, in a study at Massachusetts General Hospital and Harvard Medical School, Boston. Of the boys with autism, 16 were language impaired (ALI) and 6 had normal language ability (ALN). Their ages ranged from 6.2 to 13.4 years; they were all right-handed. As predicted, MRI brain scans showed group differences in volumetric asymmetry in language-related regions in inferior lateral frontal (Broca's area) and posterior superior temporal cortex. Language impaired boys with autism (ALI) and boys with SLI both had significant reversal of asymmetry in frontal language-related cortex. Language-related areas were larger on the right side in both ALI and SLI groups and larger on the left in both normal language groups. The boys with unimpaired language and autism (ALN) had similar asymmetry to that of control groups. Broca's area asymmetry reversal is correlated more with language impairment than with autism. The findings strengthen a proposed phenotypic link between ALI and SLI in boys. (De Fosse L, Hodge SM, Makris N, et al. Language-association cortex asymmetry in autism and specific language impairment. Ann Neurol December 2004;56:757-766). (Gordon J Harris PhD, RAD CADx LAB, MGH, Zero Emerson Place \#3A, Boston, MA 02114).

COMMENT. The abnormal asymmetry in language-related brain areas in boys with specific language impairment (SLI) or language impairment and autism is more closely related to language impairment than to autism. The authors conclude that their findings support the hypothesis of a common neurobiological basis of language impairment in autism and SLI. Language function is variable but is often impaired in autism. Autistic children with impaired language have a similar profile of language impairment to that of SLI, and a common genetic linkage is likely (Kjelgaard MM, Tager-Flusberg H, 2001). 
Developmental stuttering is associated with atypical planum temporale asymmetry, in a study by Foundas AL et al, 2001, and cited in an editorial (Foundas AL. Brain lumps and bumps: a neural risk for autism Ann Neurol Dec 2004;56:755-756). Cortical structures implicated in language deficits are not smaller but larger, and "bigger is not always better." Foundas proposes that the alterations in cellular morphology may affect a single cortical layer or may be more extensive and associated with minor heterotopias. The relation between brain morphology, language, and genetics is a potentially important area of research in autism and SLI.

Discordant mental and physical efforts in autism. Ming X, et al (Brain Dev Dec 2004;26:519-524) monitor brainstem autonomic function to detect mental effort in a child with autism and non-compliance. The patient showed decreases in cardiac vagal tone and cardiac sensitivity to baroreflex, and sustained increases of mean arterial blood pressure and heart rate concurrently, indicating an appropriate autonomic response to a mental effort but failed physical effort. The discordant mental and physical efforts indicate that the auistic patient does comply with commands mentally, and attempts to modify behavior through medications or behavioral intervention may be ill-advised.

Children's Communication Checklist to differentiate autism and ADHD. Compared to normal controls, children with High Functioning Autism showed language deficits on all CCC scales, and the information obtained on the CCC was different for ADHD and autism patients. Information obtained from both parent and teacher increased patient identification compared to that of parent alone. (Geurts HM, et al. Can the Children's Communication Checklist differentiate between children with autism, children with ADHD, and normal controls? J Child Psychology and Psychiatry Nov 2004;45:1437-1453).

\section{MOVEMENT DISORDERS}

\section{CLINICAL FEATURES OF RESTLESS LEGS SYNDROME}

Clinical characteristics of childhood-onset restless legs syndrome (RLS) were studied in $32(5.9 \%)$ patients, $<18$ years of age, diagnosed with the disorder among 538 who attended the Pediatric Sleep Disorders Center with sleep-wake complaints, between January 2000 and March 2004, at the Mayo Clinic, Rochester, MN. Subjects with RLS were classified as probable $(9 / 32[28 \%])$ or definite $(23 / 32$ [72\%]). The age of diagnosis was earlier in the probable group (11.3 years; range 6-15) than the definite group (13.9 years; range $5-17 ; \mathrm{p}=0.04)$, but otherwise, no differences were found between the two groups. All patients met the diagnostic criteria for RLS as defined by the National Institutes of Health workshop (Allen RP, et al. Sleep Med 2003;4:101-119). These include an urge to move the legs, accompanied by unpleasant sensation in the legs, worsened by periods of inactivity, relieved by movement, occurring mainly at night, complicated by sleep disturbance, and a positive family history. The average duration of follow-up was 12.3 months (range 1-44). Sleep onset or sleep maintenance insomnia was the most common complaint occurring in 28 of $32(87.5 \%)$ patients, inattentiveness was present in $25 \%$, limb movements observed during sleep as a presenting symptom in $31 \%$, chronic fatigue in $28 \%$, and sleep walking in $9.3 \%$. A 